PROCEEDINGS OF THE

AMERICAN MATHEMATICAL SOCIETY

Volume 132, Number 11, Pages 3269-3273

S 0002-9939(04)07423-4

Article electronically published on June 2, 2004

\title{
ON PERTURBATIONS OF THE GROUP OF SHIFTS ON THE LINE BY UNITARY COCYCLES
}

\author{
G. G. AMOSOV AND A. D. BARANOV \\ (Communicated by Joseph A. Ball)
}

\begin{abstract}
It is shown that the class of perturbations of the semigroup of shifts on $L^{2}\left(\mathbb{R}_{+}\right)$by unitary cocycles $V$ with the property $V_{t}-I \in s_{2}, t \geq 0$ (where $s_{2}$ is the Hilbert-Schmidt class) contains strongly continuous semigroups of isometric operators, whose unitary parts possess spectral decompositions with the measure being singular with respect to the Lebesgue measure. Thus, we describe also the subclass of strongly continuous groups of unitary operators that are perturbations of the group of shifts on $L^{2}(\mathbb{R})$ by Markovian cocycles $W$ with the property $W_{t}-I \in s_{2}, t \in \mathbb{R}$.
\end{abstract}

\section{INTRODUCTION}

Consider the one-parameter group of shifts $S=\left\{S_{t}, t \in \mathbb{R}\right\}$ acting on the Hilbert space $H=L^{2}(\mathbb{R})$ by the formula $\left(S_{t} f\right)(x)=f(x+t), f \in H$. The group of unitary operators $S$ determines a group of *-automorphisms $\alpha=\left\{\alpha_{t}, t \in \mathbb{R}\right\}$ acting on the algebra $B(H)$ consisting of all bounded operators on $H$ such that $\alpha_{t}(x)=S_{t} x S_{-t}, x \in B(H), t \in \mathbb{R}$. Let $H_{0}=L^{2}\left(\mathbb{R}_{+}\right)$and assume that $H_{0}$ is naturally embedded into $H$. Then, given $t \geq 0$, it is possible to define correctly the restrictions $T_{t}=\left.S_{-t}\right|_{H_{0}}$ and $\beta_{t}=\left.\alpha_{-t}\right|_{B\left(H_{0}\right)}$ forming the semigroup of right shifts on the semi-line $T=\left\{T_{t}, t \in \mathbb{R}_{+}\right\}$and the semigroup $\beta=\left\{\beta_{t}, t \in \mathbb{R}_{+}\right\}$ consisting of $*$-endomorphisms of $B\left(H_{0}\right)$. As shown in [1], $\beta$ satisfies the property $\bigcap_{n \in \mathbb{N}} \beta_{t n}\left(B\left(H_{0}\right)\right)=\{\mathbb{C} 1\}, t>0$. The semigroup $\beta$ is said to be the flow of Powers shifts.

Let $\mathcal{U}(H)$ and $\mathcal{U}\left(H_{0}\right)$ denote the groups of all unitary operators on the spaces $H$ and $H_{0}$. A set $W=\left\{W_{t} \in \mathcal{U}(H), t \in \mathbb{R}\right\}, W_{0}=I$, is said to be a multiplicative unitary 1-cocycle of the group $S$ (or, equivalently, of the group $\alpha$ ), if

$$
W_{t+s}=W_{t} \alpha_{t}\left(W_{s}\right), t, s \in \mathbb{R} .
$$

It follows from (1) and the condition $W_{0}=I$ that

$$
W_{-t}=\alpha_{-t}\left(W_{t}^{*}\right), t \in \mathbb{R}
$$

Received by the editors March 31, 2003 and, in revised form, July 8, 2003.

2000 Mathematics Subject Classification. Primary 47D03; Secondary 46L55, 46L57.

Key words and phrases. Group of shifts, unitary cocycles, cocycle conjugacy.

The first author is partially supported by INTAS-00-738.

The second author is partially supported by RFBR Grant No. 03-01-00377. 
Thus, it suffices to determine the cocycle only for positive or only for negative values of the parameter $t$. Analogously, it is possible to define a multiplicative unitary 1cocycle $V=\left\{V_{t} \in \mathcal{U}\left(H_{0}\right), t \in \mathbb{R}_{+}\right\}, V_{0}=I$, of the semigroup $T$ (or, what is the same, $\beta$ ) consisting of the operators $V_{t}$ satisfying the property

$$
V_{t+s}=V_{t} \beta_{t}\left(V_{s}\right), t, s \geq 0 .
$$

For short, usually one refers to $\alpha$-cocycles and $\beta$-cocycles if one needs $W$ and $V$ respectively.

We say that a semigroup of *-endomorphisms $\tilde{\beta}$ is a cocycle perturbation of the flow of Powers shifts $\beta$ if there is a $\beta$-cocycle $V$ such that

$$
\tilde{\beta}_{t}(x)=V_{t} \beta_{t}(x) V_{t}^{*}, t \geq 0, x \in B\left(H_{0}\right) .
$$

This notion was introduced by W. Arveson [2]. In the same paper it was proved that the so-called completely spatial semigroups of endomorphisms are cocycle perturbations of the flow of Powers shifts. On the other hand, already in [1] the problem was posed to investigate cocycle perturbations of the flow of Powers shifts on arbitrary von Neumann factors that are not isomorphic to the algebra $B(H)$ of all bounded operators. In the present paper we continue the investigation of cocycle perturbations initiated in [1, 2]. It is shown in [3] (and in [4], where the group of shifts with the discrete parameter $t \in \mathbb{Z}$ is considered) that, for quasifree semigroups on the hyperfinite von Neumann factors, to solve this problem one needs to describe the set of all $\beta$-cocycles $V$ with the property

$$
V_{t}-I \in s_{2}, t \geq 0
$$

where $s_{2}$ denotes the Hilbert-Schmidt class of operators. Here we give new examples of such cocycles $V$.

Note that each $\beta$-cocycle $V_{t} \in \mathcal{U}\left(H_{0}\right)$ can be extended to an $\alpha$-cocycle $W_{t} \in \mathcal{U}(H)$ if one continues the action of each $V_{t}$ by the formula $V_{t} f=f, f \in H \ominus H_{0}$, and puts $W_{-t}=V_{t}, t \geq 0$. Furthermore, for positive values of $t$ the action of the cocycle $W_{t}$ is determined by means of the equality (2). Denote by $H_{t} \subset H$ the subspace of the past before the moment $t$, which is generated by functions with supports in the segment $[-t,+\infty)$. Note that $H_{t}=S_{t} H_{0}, t \in \mathbb{R}$. The cocycle $W$ obtained by the extension procedure of $V$ given above satisfies the property of "localization of the action to the past" $\left.W_{t}\right|_{H_{t}^{\perp}}=I$ and is said to be Markovian (see [5, 6]). We shall say that the Markovian $\alpha$-cocycle $W$ and the $\beta$-cocycle $V$ are associated. Thus, to describe all $\beta$-cocycles $V$ with the property $V_{t}-I \in s_{2}, t \geq 0$, one needs to describe all Markovian $\alpha$-cocycles $W$ with the property $W_{t}-I \in s_{2}, t \in \mathbb{R}$.

In [5, 6] a model is constructed describing all Markovian $\alpha$-cocycles. The Markovian $\alpha$-cocycles are completely determined by the $\beta$-cocycles associated with them. Every $\beta$-cocycle $V$ defines a semigroup of isometric operators

$$
\tilde{T}=\left\{\tilde{T}_{t}=V_{t} T_{t}, t \in \mathbb{R}_{+}\right\} .
$$

It turns out (see [6]) that the deficiency index of the generator of $\tilde{T}_{t}$ equals one and, vice versa, every semigroup with unital deficiency index of the generator is a cocycle perturbation of $T$. Let $H_{0}=H_{u} \oplus H_{i s}$ be the Wold decomposition of the semigroup $\tilde{T}$. Here the subspaces $H_{u}$ and $H_{i s}$ reduce $\tilde{T}$ to the semigroups of unitary operators and completely non-unitary operators respectively. The techniques introduced in [3]- [7] allow one to prove (see [5, 3]) that for every unitary semigroup $U=\left\{U_{t}, t \in\right.$ $\mathbb{R}\}$ on the Hilbert space $K$ it is possible to choose a $\beta$-cocycle $V$ with $V_{t}-I \in s_{2}$ 
such that the cocycle perturbation of $T$ by $V$ leads to the semigroup $\tilde{T}$, whose unitary part $\left.\tilde{T}\right|_{H_{u}}$ is unitarily equivalent to $U$ if $U$ has a pure point spectrum or a bounded generator. In the present paper, using the results of [8], we prove that the class of perturbations of the semigroup of shifts $T$ by the $\beta$-cocycles $V$ with the property $V_{t}-I \in s_{2}, t \geq 0$, contains the semigroups of isometric operators with the unitary parts $U$, which admit the spectral representations with the measures being singular with respect to the Lebesgue measure.

\section{MAIN RESUlT}

In what follows we shall use the methods of the theory of invariant subspaces of the semigroup of shifts $T$ (see [9]). Suppose that the subspace $K \subset H_{0}$ and $T_{t} K \subset K, t \geq 0$. Then there is an inner function $\Theta$ in the upper half-plane $\mathbb{C}_{+}$(i.e., a bounded function, analytic in $\mathbb{C}_{+}$, such that the modulus of its non-tangential boundary values is equal to one a.e. with respect to the Lebesgue measure on the real line) such that $K=M_{\Theta} H_{0}$, where the isometric operator $M_{\Theta}$ is defined by the formula $M_{\Theta}=\mathcal{F}^{-1} \Theta \mathcal{F}$. Here and in what follows we denote by $\Theta$ and $\mathcal{F}$ the operator of multiplication by the function $\Theta(z)$ and the Fourier transform respectively. By $P_{L}$ we denote the orthogonal projection onto the subspace $L$.

Let $U=\left\{U_{t}, t \geq 0\right\}$ be an arbitrary strongly continuous semigroup of unitary operators on $K^{\perp}$. Define a strongly continuous semigroup of isometric operators $\tilde{T}_{t}$ on $H_{0}$ by the following formula:

$$
\tilde{T}_{t}=U_{t} P_{K^{\perp}}+T_{t} P_{K}, t \geq 0 .
$$

By means of the operator $M_{\Theta}$ one can construct a dilation of the semigroup $\tilde{T}$ to a strongly continuous group of unitary operators $\tilde{S}$ on $H$ such that

$$
\tilde{S}_{-t}=S_{-t} P_{H_{t}^{\perp}}+M_{\Theta} S_{-t} P_{H_{t} \ominus H_{0}}+\tilde{T}_{t} P_{H_{0}}, t \geq 0
$$

and for $t>0$ we should put $\tilde{S}_{t}=\tilde{S}_{-t}^{*}$. Now let us introduce the $\alpha$-cocycle $W$ by the formula $W_{t}=\tilde{S}_{t} S_{-t}, t \in \mathbb{R}$. Then, by construction, one can see that $\left.W_{t}\right|_{H_{t}^{\perp}}=I, t \geq 0$, or, what is equivalent, by virtue of the equality (2), we get $\left.W_{-t}\right|_{H_{0}^{\perp}}=I, t \geq 0$. Thus, $W$ is a Markovian cocycle, the subspace $H_{0}$ is invariant with respect to $W_{t}$ for $t \leq 0$, and it is possible to determine the $\beta$-cocycle $V_{t}=$ $\left.W_{-t}\right|_{H_{0}}, t \geq 0$ (see 6]). Notice that the semigroup $U$ is the unitary part of $\tilde{T}$. In [3] 7] it was shown that if $U$ has a pure point spectrum or a bounded generator, then there is an inner function $\Theta$ such that the cocycles $W$ and $V$ determined by the group (3) satisfy the properties $W_{t}-I \in s_{2}, t \in \mathbb{R}$, and $V_{t}-I \in s_{2}, t \geq 0$, respectively. To prove the inclusion in the Hilbert-Schmidt class, an estimation of $\Theta$ was used for $U$ with a pure point spectrum, and the von Neumann theorem was used to extend the result to $U$ with a bounded generator. In [3, 7] only $\beta$-cocycles were investigated, while in [5, 6] the notion of Markovianity was involved.

Suppose that for a strongly continuous group of unitary operators $\tilde{U}$ on a Hilbert space $\tilde{K}$ there exists a measure $\mu$ on the real line $\mathbb{R}, \mu(\mathbb{R})<+\infty$, such that $\tilde{U}$ is unitarily equivalent to the group of unitary operators $\bar{U}$ acting on the Hilbert space $L^{2}(\mu)$ by the formula

$$
\left(\bar{U}_{t} f\right)(x)=e^{i t x} f(x), f \in L^{2}(\mu) .
$$

The representation (4), which is said to be spectral, surely exists if there is a vector $e \in \tilde{K}$ cyclic with respect to the group $\tilde{U}=e^{i t H}$ (i.e., a vector $e$ such that for the 
generator $H$ the vectors $\left\{f(H) e \mid f \in L^{\infty}\right\}$ form a dense set in $\left.\tilde{K}\right)$. An arbitrary group of unitary operators $\tilde{U}$ is unitarily equivalent to a no more than countable orthogonal sum of unitary groups of the form (4) (see [10]). Note that a group of unitary operators possessing both bounded generator and pure point spectrum admits the spectral decomposition (4). So the following theorem gives also an alternative proof of the results obtained in [3]- 7 .

Theorem 1. Suppose $\tilde{U}$ is a strongly continuous semigroup on a Hilbert space $\tilde{K}$ that admits a spectral representation (4), where $\mu$ is singular with respect to the Lebesgue measure. Then there are an inner function $\Theta$ and a semigroup of unitary operators $U$ on the Hilbert space $K^{\perp}=H_{0} \ominus M_{\Theta} H_{0}$, which is unitarily equivalent to $\tilde{U}$, such that the Markovian $\alpha$-cocycle $W_{t}=\tilde{S}_{t} S_{-t}, t \in \mathbb{R}$, determined by the group (3) and its restriction $V_{t}=\left.W_{-t}\right|_{H_{0}}, t \geq 0$, which is a $\beta$-cocycle, satisfy the properties $W_{t}-I \in s_{2}, t \in \mathbb{R}$ and $V_{t}-I \in s_{2}, t \geq 0$.

Proof. The measure of the spectral representation $\mu$ defines the singular inner function $\Theta$ :

$$
\Theta(z)=\exp \left(i \int_{\mathbb{R}} \frac{d \mu(t)}{t-z}\right), z \in \mathbb{C}_{+} .
$$

It is shown in 8 that the condition $\mu(\mathbb{R})<+\infty$ implies that

$$
1-\Theta \in H^{2}\left(\mathbb{C}_{+}\right) .
$$

Essentially, this is a half-plane version of the results of P. R. Ahern and D. N. Clark [11] for the unit disk. The Ahern-Clark theorem states that if an inner function $\theta$ in the unit disk has a finite angular derivative at a point $\zeta,|\zeta|=1$, then the nontangential boundary value $\theta(\zeta)$ is well-defined and the function

$$
g_{\zeta}(z)=\frac{1-\overline{\theta(\zeta)} \theta(z)}{1-\bar{\zeta} z}
$$

belongs to the Hardy class $H^{2}$ in the disk. Now the inclusion (5) follows by means of a conformal mapping of the unit disk onto the half-plane.

It follows from (5) that the operator $(1-\Theta) \mathcal{F}: L^{2}([0, t]) \rightarrow H$ belongs to the Hilbert-Schmidt class $s_{2}$, since its kernel $k(x, y)=(1-\Theta(y)) e^{-i x y}$ satisfies the condition $k \in L^{2}([0, t] \times \mathbb{R})$. Thus,

$$
P_{H_{0} \ominus H_{-t}} M_{\Theta} P_{H_{0} \ominus H_{-t}}-P_{H_{0} \ominus H_{-t}} \in s_{2}, t \geq 0 .
$$

By virtue of the theorem on triangulation of the truncated shift (see [9]), the group $\bar{U}$ defined by the formula (4) is unitarily equivalent to a certain group $U=\left\{U_{t} \mid t \in\right.$ $\mathbb{R}\}$ acting on the space $K^{\perp}$ such that

$$
U_{t}=\left.P_{K^{\perp}} T_{t}\right|_{K^{\perp}}+R, R \in s_{2} .
$$

Thus, the operator $U_{t}$ is a normal part of the operator of the truncated shift $\left.P_{K^{\perp}} T_{t}\right|_{K^{\perp}}$.

Let us define the operators $\tilde{S}_{t}$ by (3). Then

$$
\tilde{S}_{-t}-S_{-t}=\left(T_{t}-U_{t}\right) P_{K^{\perp}}+\left(M_{\Theta}-I\right) S_{-t} P_{H_{t} \ominus H_{0}} .
$$

Considering the cocycle $W_{t}=\tilde{S}_{t} S_{-t}$, we get that (8) is equivalent to

$$
W_{-t}-I=\left(T_{t}-U_{t}\right) P_{K^{\perp}} S_{t} P_{H_{-t}}+\left(M_{\Theta}-I\right) P_{H_{0} \ominus H_{-t}}, t \geq 0 .
$$


The first term in the right-hand side of (9) belongs to $s_{2}$ by virtue of the theorem on triangulation (7). The second term is in $s_{2}$ due to (6).

The authors would like to thank K. M. Dyakonov for a careful reading of the text.

\section{REFERENCES}

1. R. T. Powers, An index theory for ${ }^{*}$-semigroups of endomorphisms of $\mathcal{B}(\mathcal{H})$ and type $\mathrm{II}_{1}$ factors, Canad. J. Math. 40 (1988), 86-114. MR 89f:46116

2. W. Arveson, Continuous analogues of Fock space, Mem. Amer. Math. Soc. 409 (1989), 1-66. MR 90f:47061

3. G. G. Amosov, On cocycle conjugacy of quasifree endomorphism semigroups on the CAR algebra, J. Math. Sci. (New York) 105 (2001), 2496-2503. MR 2003d:46088

4. G. G. Amosov, Approximation modulo $s_{2}$ of isometric operators and cocycle conjugacy of endomorphisms of the CAR algebra, Fundamental. i Prikl. Matem. 7 (2001), 925-930 (Russian). MR 2002j:46078

5. G. G. Amosov, Cocycle perturbation of quasifree algebraic K-flow leads to required asymptotic dynamics of associated completely positive semigroup, Infin. Dimens. Anal. Quantum Probab. Relat. Top. 3 (2000), 237-246. MR 2001m:46148

6. G. G. Amosov, Stationary quantum stochastic processes from the cohomological point of view, Quantum Probability and White Noise Analysis XV, Edited by W. Freudenberg, World Sci. Publ. Co. River Edge, NJ, 2003, 29-40.

7. G. G. Amosov, On the approximation of semigroups of isometries in a Hilbert space, Russian Math. (IzVUZ) 44 (2000), no. 2, 5-10. MR 2001f:47066

8. A. D. Baranov, Isometric embeddings of the spaces $K_{\Theta}$ in the upper half-plane, J. Math. Sci. (New York) 105 (2001), 2319-2329. MR 2002h:46039

9. N. K. Nikolski, Treatise on the shift operator, Springer-Verlag, 1986.

10. N. Dunford and J. T. Schwartz, Linear operators. Part II. Spectral theory, John Wiley \& Sons, 1988. MR 90g:47001b

11. P. R. Ahern and D. N. Clark, Radial limits and invariant subspaces, Amer. J. Math., 92 (1970), 332-342. MR 41:7117

Department of Higher Mathematics, Moscow Institute of Physics and Technology, Dolgoprudni 141700, Russia

E-mail address: amosov@fizteh.ru

Department of Mathematics and Mechanics, St. Petersburg State University, 28, Universitetski Pr., St. Petersburg, 198504, Russia

Current address: Laboratoire de Mathématiques Pures, Université Bordeaux 1, 351, Cours de la Libération, 33405 Talence, France

E-mail address: antonbaranov@netscape.net 